\title{
Distinguishing between the precision of attentional localization and attentional resolution
}

\author{
Cathleen M. Moore \\ University of Iowa, Iowa City, Iowa \\ LYNDSEY K. LANAGAN-LeITZEL \\ Pennsylvania State University, University Park, Pennsylvania \\ AND \\ Elisabeth M. Fine \\ Schepens Eye Research Institute, Boston, Massachusetts
}

\begin{abstract}
Attentional resolution is a construct that refers to the minimal separation that allows one stimulus to be attended separately from nearby stimuli. The attentional walk task, which requires a series of covert shifts of attention within variably dense arrays of stimuli, was introduced as a method of measuring attentional resolution. Using a cuing task, we show that individual items within arrays that are too dense to support an attentional walk can nonetheless be attended. We note that the precision with which attention can be localized is, in principle, a limitation separate from attentional resolution and conclude that performance in the attentional walk task is better suited for measuring this limitation than for measuring attentional resolution per se.
\end{abstract}

Numerous metaphors have been used to discuss visual attention (see Fernandez-Duque \& Johnson, 1999, for a review). These include visual attention as a spotlight (e.g., Posner, 1980; Posner, Snyder, \& Davidson, 1980), as a zoom lens (e.g., Eriksen \& St. James, 1986; Eriksen \& Yeh, 1985), as a gradient filter (e.g., LaBerge \& Brown, 1989), and as a nonlinear filter (e.g., Cutzu \& Tsotsos, 2003; Tsotsos et al., 1995). All of these metaphors characterize mechanisms by which visual information can be processed selectively. They also share the property of attributing a spatial extent to the area over which visual attention can be focused. Reflecting this property, researchers have asked about limitations of the spatial extent of focused attention; specifically, how narrowly can attention be focused and how does it vary across conditions, such as location within the visual field?

This question, which has been characterized as the question of attentional resolution (He, Cavanagh, \& Intriligator, 1996, 1997; Intriligator \& Cavanagh, 2001), has been addressed in a variety of ways. For example, using the flankers task, in which observers identify a central target letter that is flanked by distractor letters, Eriksen and Hoffman (1972) estimated the extent of selection to be about $1^{\circ}$ of visual angle. Others, using probe detection and related methods, have proposed a gradient area of selection extending as much as $19^{\circ}$ from a cued location (e.g., Downing \& Pinker, 1985; Henderson \& Macquistan, 1993; LaBerge \& Brown, 1986).
Still others, using methods involving many distractors and, sometimes, multiple targets separated by variable distances, have suggested that the area of selection has a facilitatory-center-inhibitory-surround profile with a spatial extent that is as large as $6^{\circ}$ and as small as $1^{\circ}$ (e.g., Bahcall \& Kowler, 1999; Cutzu \& Tsotsos, 2003; Mounts, 2000a, 2000b; Steinman, Steinman, \& Lehmkuhle, 1995). Finally, others have suggested that the spatial extent and shape of selection varies with the task and characteristics of the stimuli (e.g., Eriksen \& St. James, 1986; Juola, Bouwhuis, Cooper, \& Warner, 1991; LaBerge, 1983; LaBerge \& Brown, 1986), as well as with the location of stimuli within the visual field (e.g., He et al., 1996, 1997; Sagi \& Julesz, 1986).

Citing concern regarding the heterogeneity of the approaches and findings, Intriligator and Cavanagh (2001) introduced the attentional walk task as a method for measuring attentional resolution. In this task, observers are shown arrays of identical disks similar to those shown in Figure 1 that vary in density and eccentricity. Observers fixate the central point, and a short time later, one of the disks briefly changes color, indicating that attention (but not fixation) should be shifted to that disk. Following this cue, a series of instructions is given directing the observer to shift attention one disk to the left or to the right of the currently attended disk (e.g., "left," "left," "right," "left," etc.). At the end of a five- to seven-step series of commands, observers are probed with another 


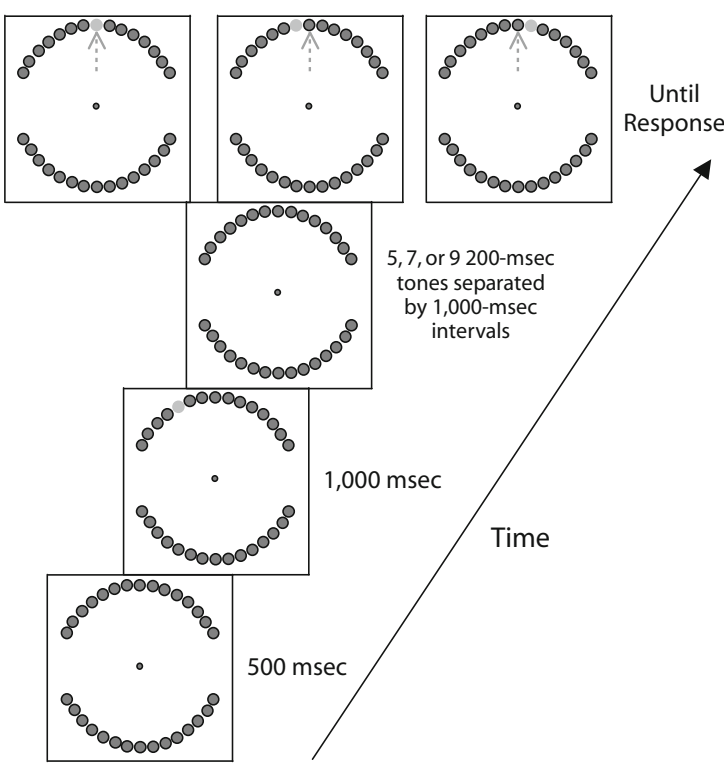

Figure 1. Experiment 1 trial events. Trials began with a fixation display. The subjects were asked to remain fixated on the central dot throughout the trial. A cue indicated the start disk for the attentional walk. This was followed by a series of five, seven, or nine high- and low-pitched tones indicating a shift of attention to the right or left, respectively. The tones were followed with a probe location indicated by one of the disks changing color. The top row illustrates alternative response displays for the following example series of directions: right, right, right, left, right. The small arrows in these displays were not present during the experiment. They are shown here to indicate the correct end of the walk. Correct responses to the three displays, from left to right, would be "yes," "no," and "no."

disk that changes color. The task is to indicate whether or not the final probe disk marks the end point of the attentional walk. The logic of this task is that if attentional resolution is coarser than the distance between the disks, observers will be unable to reliably select one disk over an adjacent disk in response to the shift commands and will, therefore, be unable to reliably follow the attentional walk. The highest density within which observers are able to reliably report the endpoint of an attentional walk would then provide an estimate of the resolution of attention for those particular conditions (e.g., the eccentricity of the array and where within the visual field the walk occurred).

Using the attentional walk task, Intriligator and Cavanagh (2001) showed that there is a fairly broad range of display densities for which one can easily perceive that there are individual items, yet one cannot execute an attentional walk. Thus, there is a notable dissociation between attentional resolution and spatial acuity. In addition, they found that attentional resolution measured this way tends to be coarser in the upper visual field than in the lower visual field (see also He et al., 1996, 1997) and that it tends to be coarser with increasing eccentricity, even when one controls for differences in spatial acuity.
An advantage of the attentional walk task over previous methods of measuring the spatial extent of attention is that the items within which the selection must occur are identical. Therefore, interference in the walk task can be attributed directly to spatial limitations of visual selection. The link to spatial limitations through other tasks, such as the flankers task, is less direct because the interference that is measured can be caused by confusion over which feature belongs to which object. Thus, asking about selection within arrays of identical items, where feature ownership is irrelevant, affords a more direct measure of spatial selectivity.

In the present study, we asked whether the attentional walk task reflects only limitations of attentional resolution or whether it also reflects - perhaps even primarily reflects-limitations in the precision with which the focus of attention can be localized, however wide or narrow the selection region may be. Metaphorically, the distinction between attentional resolution and the precision of attentional localization is like the distinction between the smallest diameter to which a spotlight can be reduced versus the spatial reliability with which the center of the spotlight, however large or small, can be moved from one location to another. These are separable limitations, and both are important for characterizing the properties and limitations of spatial attention.

The demands of the attentional walk task seem well suited for capturing the limitations of the precision of attentional localization. Consider the details of the attentional walk task more closely. First, implicit in the logic of the task is the assumption that observers can allocate their attention to the start disk on the basis of the color change (see Figure 1). Thus, even though observers may be unable to reliably execute an attentional walk within an array of a given density, it is assumed that they can use the color cue to start the walk at the correct location. Note that this would require the selection of the cued disk from the surrounding disks, which would require a spatial extent within the range of the separation given by that density. In principle, failures in an attentional walk could occur because attention was never reliably allocated to the start disk, attention could not be shifted reliably within the array of items during the walk, or a combination of the two. Because these different sources of failure imply the breakdown of different mechanisms in different ways, it would be useful to be able to distinguish among them.

There is a second characteristic of the attentional walk task that must be considered in identifying what limitations it reflects. Specifically, the initial movement in the attentional walk task may be fundamentally different from the later steps in the walk. In particular, the initial shift of attention is exogenously controlled; the location toward which attention is to be directed is specified by a physical change in the display at that location. In contrast, the remaining attentional shifts are endogenously controlled; the location toward which attention is to be directed is specified without the explicit support of a change in the stimulus at that location. Therefore, assuming that atten- 
tion can be reliably allocated to the cued start disk, failures of the attentional walk after that could reflect either a failure of the spatial extent of selection for endogenously cued targets (i.e., insufficient attentional resolution) or, again, a failure to reliably focus attention on the target location, even though, when attention is focused there, the target can be selectively processed relative to its adjacent items (i.e., sufficient attentional resolution).

In the experiments reported here, we measured response time (RT) in a cuing task similar to that of Posner and colleagues (e.g., Posner et al., 1980) and found that observers were able to allocate attention, both exogenously and endogenously, within arrays of items that were too dense to support a reliable attentional walk. These results suggest that the attentional walk task does not reflect strict limitations on the spatial extent of selection. If it did, there should have been no advantage provided by cues within the highly dense arrays. We suggest that the attentional walk task is better suited for measuring the precision with which attention can be localized than for measuring attentional resolution and, more generally, that drawing a distinction between these different limitations of attention is useful both for characterizing the spatial properties of attention and for reconciling apparently heterogeneous findings. In related work, we drew this distinction more formally by adapting psychophysical models of contrast sensitivity to include the effects of visual attention (Palmer $\&$ Moore, in press).

\section{EXPERIMENT 1}

In Experiment 1, we used an attentional walk task to identify specific densities for which observers could and could not reliably execute an attentional walk. Those densities were then used to test whether attention could be allocated within these displays on the basis of exogenous (Experiments 2 and 3 ) and endogenous (Experiment 4) cues.

\section{Method}

Observers. Three members of the laboratory (one author) served as observers. All reported normal or corrected-to-normal visual acuity and color vision.

Equipment. The experiment was controlled by an IBM-compatible PC using custom software written in Turbo Pascal (Version 7.0). Stimuli were presented on a 21 -in. color monitor with a resolution of $1,024 \times 768$ and a refresh rate of $60 \mathrm{~Hz}$. Viewing distance was fixed for all the subjects by using a chinrest at $65 \mathrm{~cm}$.

Stimuli. Displays consisted of two arrays of 7,11,15, or 17 gray $\left(7.0 \mathrm{~cd} / \mathrm{m}^{2}\right)$ disks $\left(1.0^{\circ}\right.$ in diameter $)$ presented on a dark background. Each array subtended $140^{\circ}$ of an imaginary circle that was centered at $6.16^{\circ}$ eccentricity around a central $0.33^{\circ} \times 0.33^{\circ}$ white fixation cross (see Figure 1). Disks were evenly spaced within the arrays. The cue indicating the start disk and the end-of-walk probe disk was red $\left(6.5 \mathrm{~cd} / \mathrm{m}^{2}\right)$. High $(800-\mathrm{Hz})$ and low $(500-\mathrm{Hz})$ tones were used to indicate left and right steps, respectively.

Procedure and Task. Each of the observers completed three sessions on 3 separate days. The first session, which was a practice session, consisted of six blocks of 24 trials each. The later sessions consisted of a block of 24 practice trials, followed by six blocks of 24 trials each from which data were collected.

Trial events are illustrated in Figure 1. Trials began with the presentation of the central fixation cross and an array of gray disks.

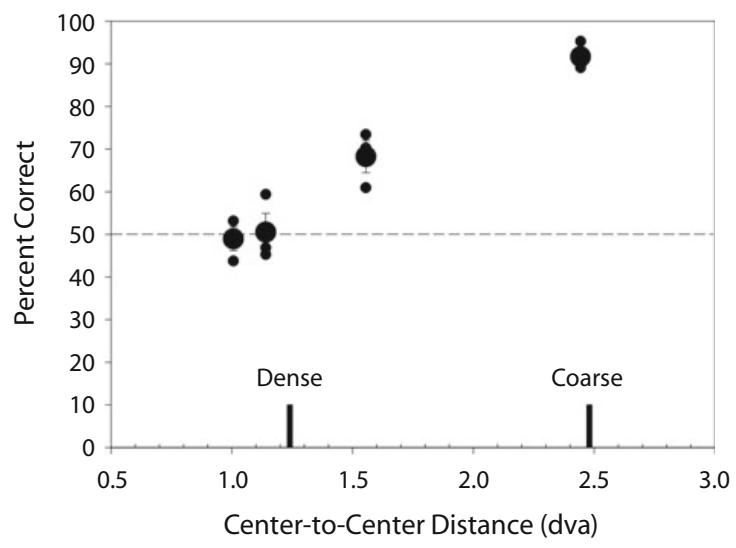

Figure 2. Mean accuracy in Experiment 1. The large symbols are the means across the subjects' data, with the error bars indicating the standard errors of the means. The small symbols are the data from individual observers. The two large tick marks on the abscissa indicate the spacings used for the dense and coarse conditions in Experiments 2-4.

The observers initiated the trial by pressing the space bar on the computer keyboard; $500 \mathrm{msec}$ later, one of the disks changed to red for $1,000 \mathrm{msec}$ and then back to gray. This was followed $1,000 \mathrm{msec}$ later by a series of five, seven, or nine $200-\mathrm{msec}$ tones separated by 1,000 -msec silent intervals. The tones were low or high, indicating a left or a right shift, respectively. Direction was random, except for constraints that forced the walk to occur entirely within the upper visual field or entirely within the lower visual field, depending on whether the start disk was in the upper or the lower visual field. One second after the final tone, a disk changed to red. This disk was the correct end point for the attentional walk, one disk to the right, or one disk to the left. The observers then responded with a right keypress ("/ " key) if they thought the red disk was the end point of their attentional walk or a left keypress (" $z$ " key) if they thought it was not. Following a 2-sec intertrial interval, during which the screen was blank, the fixation display for the next trial appeared.

The observers rested for as long as they liked between blocks of trials. They received trial-by-trial feedback as to whether their responses were correct (a visual "correct" or "incorrect") during practice blocks only.

Design. A 4 (density: 7, 11, 15, or 17 disks) $\times 3$ (number of steps: 5,7 , or 9 ) within-subjects design was used. The data were collapsed across number of steps and sessions, resulting in a total of 72 observations per data point per observer.

\section{Results and Discussion}

The results (shown in Figure 2) were similar to those reported by Intriligator and Cavanagh (2001). The large symbols indicate the mean percent correct across observers as a function of center-to-center distance between items in the display, which was determined by the density of the arrays. The smaller symbols are data from the individual observers. All the observers showed the same pattern of results; performance increased from chance levels at the smallest spacing (highest density) to near-ceiling levels at the largest spacing (lowest density) $[F(3,6)=118.35, p<$ $.001]$. The interpretation of these data is that the observers were unable to attend to the cued disk and/or reliably shift attention within the densest arrays in this experiment but 
were able to do so in the coarsest arrays. These data were used to determine the densities used in Experiments 2-4.

\section{EXPERIMENT 2}

Experiment 2 used an exogenous cuing task similar to that of Posner and colleagues (e.g., Posner et al., 1980) within circular arrays of identical items. A single item within the array was cued by a luminance change. An oriented line (the target) was then presented in the cued disk, in an adjacent disk, or in a disk two items away from the cued disk. The observers were to indicate the orientation of the line as quickly as possible. The logic was that if RT was shorter to a target in the cued disk than to a target in a disk immediately adjacent to the cued disk, it could be inferred that information in the cued disk was processed selectively and, therefore, that the spatial extent of selection was at least as fine as the separation of adjacent items in that display. In contrast, if the resolution of attention was coarser than the separation of adjacent items in that display, there should be no advantage for targets in the cued items, relative to targets that appeared one item away, although the cued location might show an advantage over targets appearing two items away. Note that this essentially tests the assumption that attention can be guided to the start disk in an attentional walk task.

Two array densities were used that were selected on the basis of the results from Experiment 1. The denser of the two had separations that would yield chance performance in an attentional walk task. The coarser of the two densities was then created by removing every other item in the dense array. This resulted in a density that had spacings that would yield near-ceiling performance in an attentional walk task. The separation values of these two densities are indicated, relative to the conditions in Experiment 1, with heavy tick marks and labels along the $x$-axis in Figure 2. Note that the targets in the one-away condition for coarse arrays were the same distance from the cue as the targets in the two-away condition for dense arrays; they differed only in whether there was an intervening item or not.

To anticipate the results, a reliable one-away cuing effect occurred even for the dense arrays. This indicates that the observers were able to process individual items selectively even within arrays that led to chance performance in the attentional walk task.

\section{Method}

Observers. Seventeen undergraduate students at the Pennsylvania State University received research credit in an introductory course in psychology for participating in the experiment. All reported normal or corrected-to-normal visual acuity.

Apparatus. Stimuli were presented on a 21-in. color monitor controlled by an IBM-compatible Pentium-based computer running custom software written in Turbo Pascal Version 7.0. Responses were recorded using a custom-built button box.

Stimuli. Displays consisted of circular arrays of identical gray disks (see Figure 3). Viewing distance was fixed at $53 \mathrm{~cm}$ using a chinrest. The arrays were presented at $6.14^{\circ}$ from fixation (measuring to the center of a disk), and each disk was $0.97^{\circ}$ in diameter. Two densities were used; coarse arrays had 9 disks per $180^{\circ}$ of arc of the circle, and dense arrays had 17 disks per $180^{\circ}$ of arc of the circle. ${ }^{1}$ The center-to-center distance for the disks was $1.24^{\circ}$ in the dense arrays

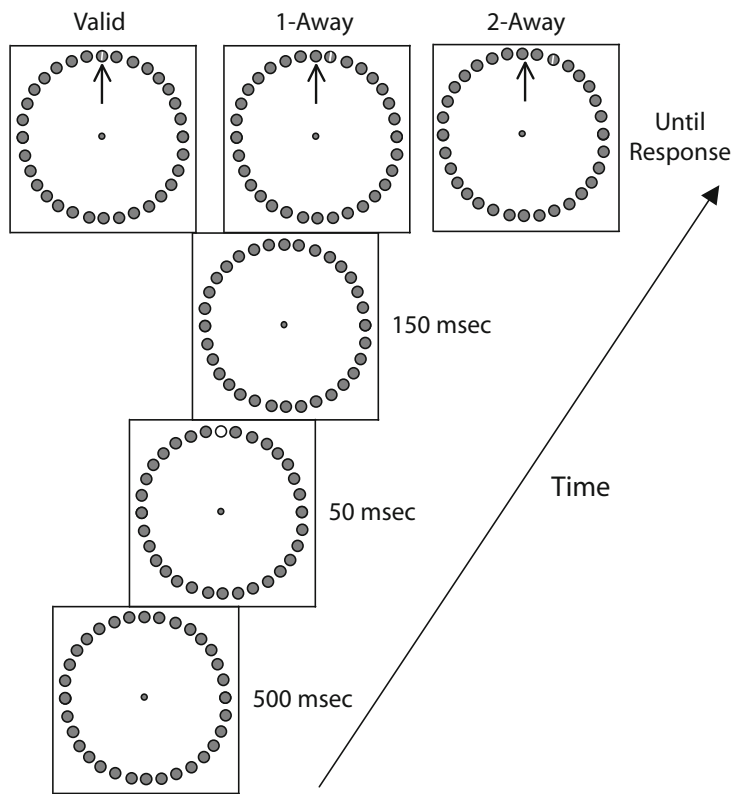

Figure 3. Experiment 2 trial events. The top row illustrates alternative response displays for the three validity conditions. The arrows in these displays were not present during the experiment. They are shown here to indicate the cued location.

and $2.48^{\circ}$ in the coarse arrays. The fixation marker was a smaller disk $\left(0.27^{\circ}\right.$ in diameter $)$ and was located at the center of the monitor, which was also the center of the array. The luminance of the gray disks was $3.0 \mathrm{~cd} / \mathrm{m}^{2}$, presented on a dark background $\left(0.13 \mathrm{~cd} / \mathrm{m}^{2}\right)$. The cue was a change in luminance of one of the gray disks $\left(51 \mathrm{~cd} / \mathrm{m}^{2}\right)$. Targets were single-pixel-wide white lines of the same luminance as the cue. Their length was somewhat smaller than the diameter of the disks $\left(0.65^{\circ}\right)$. Each line was centered in the disk and oriented $7^{\circ}$ to the left or to the right of vertical.

Task. The task was to report the orientation of the line (left or right) that appeared in one of the gray disks by pressing either the left or the right button, respectively, on the button box. The subjects were encouraged to make their responses as quickly as possible while maintaining at least a $95 \%$ level of accuracy.

Design. A 2 (density: dense or coarse) $\times 3$ (validity: valid, oneaway, or two-away) within-subjects design was used. Validity was defined by the relationship between the location of the cue and the location of the target. The cue was chosen randomly from among all of the disks above and below the horizontal meridian. The cue was never one of the two disks that appeared directly on the horizontal meridian (i.e., in the 3 o'clock or 9 o'clock position). Valid trials were those on which the target appeared at the cued location. Oneaway and two-away trials were ones on which the target appeared one item or two items away from the cued item, respectively. Valid, one-away, and two-away trials were all equally likely; one third of the targets appeared at the cued location, one third appeared one item away (half in the clockwise direction and half in the counterclockwise direction), and one third appeared two items away (again, half in the clockwise direction and half in the counterclockwise direction). The direction for the one-away and two-away trials was chosen randomly for each trial, and data were collapsed across direction for analysis. Finally, the target was oriented to the right on half the trials and to left on the other half. The data were collapsed across target orientation for analysis. Each of the 12 experimental conditions occurred equally often in a pseudorandom order within the eight 48-trial blocks. Trials on which an error was made were 
repeated at a random point later in the block, and a random recovery trial was presented following each error. The data from these trials were not included in the analyses. This resulted in 64 observations in each condition for each observer.

Procedure. Each observer completed a single session, which lasted approximately $50 \mathrm{~min}$. A session began with a set of written instructions presented on the computer that described the task. The instructions emphasized that the observers should maintain fixation on the central dot throughout the trial, but otherwise their eye movements were not controlled or monitored. After reading the instructions, the observers completed a 24-trial block of practice trials, followed by the eight 48 -trial experimental blocks. A break was provided between blocks, during which the mean RT and accuracy from the preceding block was displayed. The observers initiated the next block at their own pace.

Trial events are illustrated in Figure 3. Trials began with the presentation of the fixation point and the array of gray disks. The central fixation point remained on the screen throughout the trial. A 50-msec cue (one disk changing to white) was presented $500 \mathrm{msec}$ following the onset of the fixation display. The target was presented $150 \mathrm{msec}$ following cue offset. The target display remained on the screen until a response was recorded. When a wrong key was pressed, the word "incorrect" was presented on the screen for $500 \mathrm{msec}$ before continuing to the next trial. There was a $1,500-\mathrm{msec}$ intertrial interval, during which the screen was blank.

\section{Results and Discussion}

Mean RTs are shown in Figure 4, and error rates (ERs) are given in Table 1. Mean RTs for individual observers were submitted to a 2 (density) $\times 3$ (validity) ANOVA. There were reliable main effects of validity $[F(2,32)=$ $46.16, p<.001]$ and density $[F(1,16)=6.95, p<.05]$. There was also a significant interaction between density and validity $[F(2,32)=13.5, p<.001]$.

Planned comparisons confirmed that there was a reliable one-away cost (i.e., one-away RT - valid RT) for both the dense- and the coarse-array conditions [dense, $43 \mathrm{msec}, t(16)=7.07, p<.01$; coarse, $96 \mathrm{msec}, t(16)=$ $7.9, p<.01]$. The additional cost of being two items away from the cue (i.e., two-away RT minus one-away RT) was significant for the dense arrays $[25 \mathrm{msec} ; t(16)=2.34$, $p<.05$ ], but not for the coarse arrays [23 msec; $t(16)=$

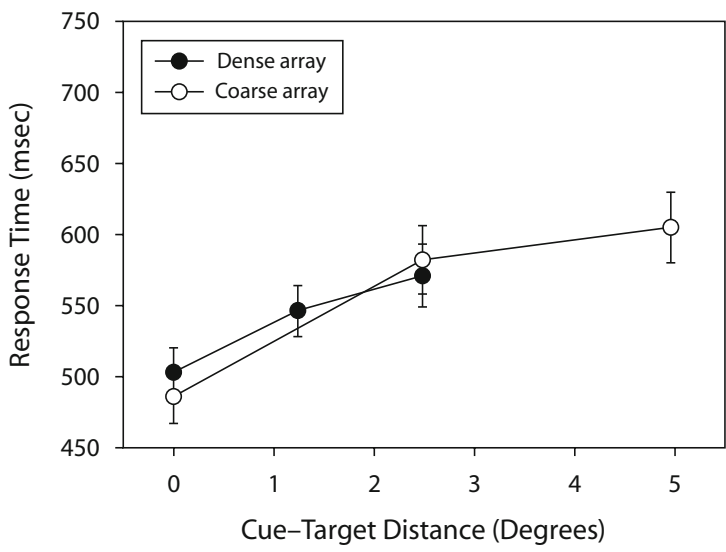

Figure 4. Mean response times in Experiment 2 shown as a function of cue-to-target distance. Error bars indicate the standard errors of the means.
Table 1

Mean Error Rates for Experiments 2 and 3 (With Standard Errors)

\begin{tabular}{|c|c|c|c|c|c|c|}
\hline \multirow[b]{3}{*}{ Condition } & \multicolumn{6}{|c|}{ Validity } \\
\hline & \multicolumn{2}{|c|}{ Valid } & \multicolumn{2}{|c|}{ One-Away } & \multicolumn{2}{|c|}{ Two-Away } \\
\hline & $M$ & SEM & $M$ & $S E M$ & $M$ & SEM \\
\hline \multicolumn{7}{|c|}{ Experiment 2} \\
\hline Coarse array & 6.1 & 1.6 & 4.8 & 1.7 & 7.0 & 1.3 \\
\hline Dense array & 5.5 & 1.3 & 5.5 & 1.1 & 5.9 & 1.5 \\
\hline \multicolumn{7}{|c|}{ Experiment 3} \\
\hline Dense disks & 8.7 & 1.6 & 9.3 & 1.8 & & \\
\hline Annulus & 7.6 & 1.6 & 8.8 & 1.6 & & \\
\hline \multicolumn{7}{|c|}{ Experiment 4} \\
\hline Coarse array & 4.9 & 1.2 & 4.4 & 1.2 & & \\
\hline Dense array & 5.7 & 1.3 & 7.7 & 1.7 & & \\
\hline
\end{tabular}

1.8, n.s.], despite the numeric similarity in the observed effects. Finally, the difference between valid and two-away in the dense condition (mean difference $=68 \mathrm{msec}$ ) was compared with the difference between valid and one-away in the coarse condition (mean difference $=96 \mathrm{msec}$ ). These conditions were identical in terms of the spatial distance between the valid and the invalid conditions; they differed only in that there was an intervening item between the two in the dense array, but not in the coarse array. The 28 -msec difference between these two effects was reliable $[t(16)=$ $2.5, p<.05]$. This contrast could indicate that there is more to the limitation of selection than just spatial separation, or it could reflect greater lateral contour interference in the dense arrays than in the coarse arrays.

All of the same analyses were conducted on the ERs. There were no significant effects that were in a direction different from that found in the analysis of RTs, indicating that the pattern of results was not contaminated by speedaccuracy trade-offs. ${ }^{2}$

The results show that attention can be allocated to an individual item, relative to items immediately adjacent to them, even for high-density arrays that would not support a successful attentional walk. This follows because there was a reliable one-away cuing effect for both the coarse and the dense arrays. Had the dense arrays been too dense for observers to reliably allocate attention within them, as chance performance in the attentional walk task suggests, targets in the cued item should not have sustained an advantage over targets in items immediately adjacent to them. This provides support for the assumption that attention can be allocated to the exogenously cued start disk in the attentional walk task and that failures in the walk task reflect limitations in the precision with which attention can be localized, rather than (only) the spatial resolution of attention.

A question regarding this experiment is whether the disks in the array played any role at all in limiting the allocation of attention as measured by the cuing effect. That is, the observers may simply have attended to the explicitly indicated location of the exogenous cue, and the presence of the individual disks - densely packed or not-was irrelevant. If this were the case, it would limit the generality of these findings and, in particular, limit the extent to which the results from the cuing task are 
comparable to results from the attentional walk task, wherein the individual disks clearly play an important role.

\section{EXPERIMENT 3}

To test the possibility that the disks were irrelevant to the cuing effects that were observed in Experiment 2, we asked whether a similar effect would occur within a solid, undifferentiated annulus that subtended the same area as the dense array of disks (see Figure 5). If an exogenous cue within an undifferentiated annulus yielded the same effect as a dense array of disks, it would suggest that the disks themselves were irrelevant in Experiment 1 , and it would limit our ability to compare those results with the results obtained in the attentional walk task. If, however, the cuing effect was smeared in the annulus condition, it would support the conclusion that the disks were relevant and that the cuing effect reflected an ability to attend to one disk distinctly from another in these dense arrays. By smeared, we mean that the cuing effect for a distance within the annulus that was equivalent to that of a one-away item in a dense array of disks is attenuated or eliminated. To anticipate the results, the cuing effect was smeared in the annulus condition, supporting the initial interpretation of the cuing effects in Experiment 2.

\section{Method}

Except where noted here, the method was the same as that in Experiment 2.

Observers. Twenty individuals from the same pool as that in Experiment 2 were tested. None had participated in Experiment 2, and all reported normal or corrected-to-normal visual acuity.

Stimuli. The array condition used displays like those in the densearray condition in Experiment 2. The annulus condition used an annulus whose inner and outer borders were $5.65^{\circ}$ and $6.62^{\circ}$ from fixation, respectively, spanning the same distance as the diameter of the disks. The annulus was solid and was the same gray as the disks in the array condition. The cue and target were the same as those in Experiment 2 and were the same across the array and annulus conditions.

Task. The task was the same as that in Experiment 2.

Design. A 2 (display: array or annulus) $\times 2$ (validity: valid or oneaway) within-subjects design was used. Note that only two validity conditions were used, valid and one-away. This was done to reduce the design and increase the number of observations per condition.

Procedure. The general procedure and specific trial events were the same as those in Experiment 2.

\section{Results and Discussion}

Mean RTs are shown in Figure 6 as a function of the spatial separation between the cue and the target. ERs are given in Table 1. The RT data were submitted to a 2 (display) $\times 2$ (validity) ANOVA. There were reliable main effects of display $[F(1,19)=20.15, p<.001]$ and validity $[F(1,19)=51.09, p<.001]$. There was also a significant interaction between display and validity $[F(1,19)=24.81$, $p<.001]$.

Planned comparisons confirmed that there was a reliable cuing effect for both display conditions [disk array, $58 \mathrm{msec}, t(19)=7.9, p<.01$; annulus, $16 \mathrm{msec}, t(19)=$ $2.7, p<.05]$. Although there was a cuing effect in both

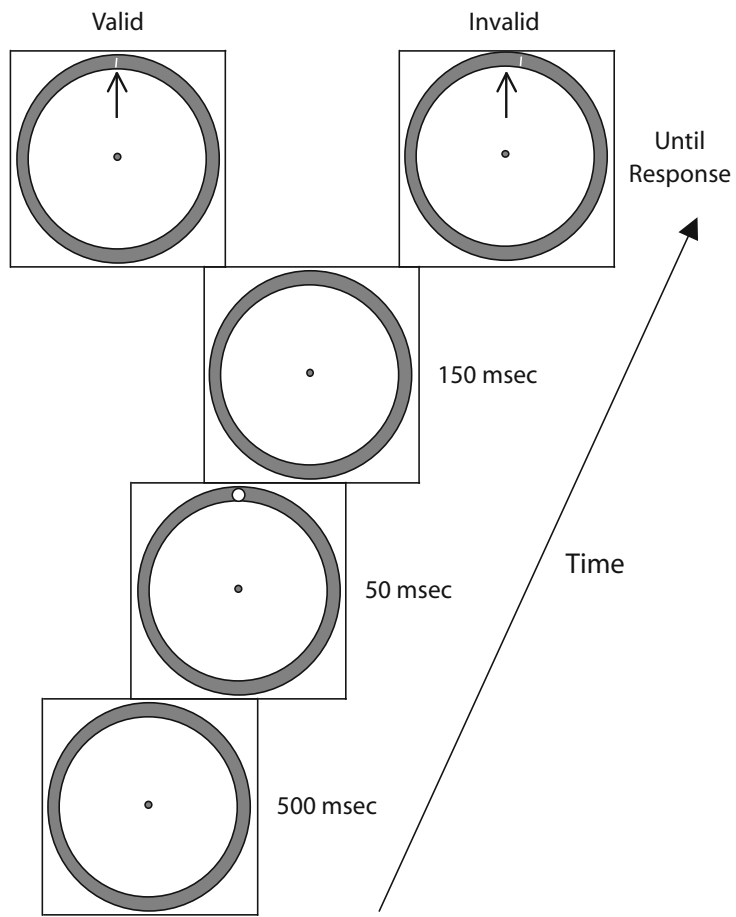

Figure 5. Experiment 3 trial events shown for the annulus condition only. (Trials in the array condition used displays similar to those in the dense condition of Experiment 2.) Trials in the annulus condition used a solid annulus. Only two validity conditions were used. The top row illustrates alternative response displays for the two validity conditions in the annulus condition; invalid in this experiment was equivalent to one-away in Experiment 2 in terms of distance. The small arrows inside the rings indicate the cue locations for the purposes of this figure; they did not appear in the trial displays.

conditions, the significant interaction confirms that the difference in magnitude of these effects was reliable. A post hoc comparison indicated that RT in the valid con-

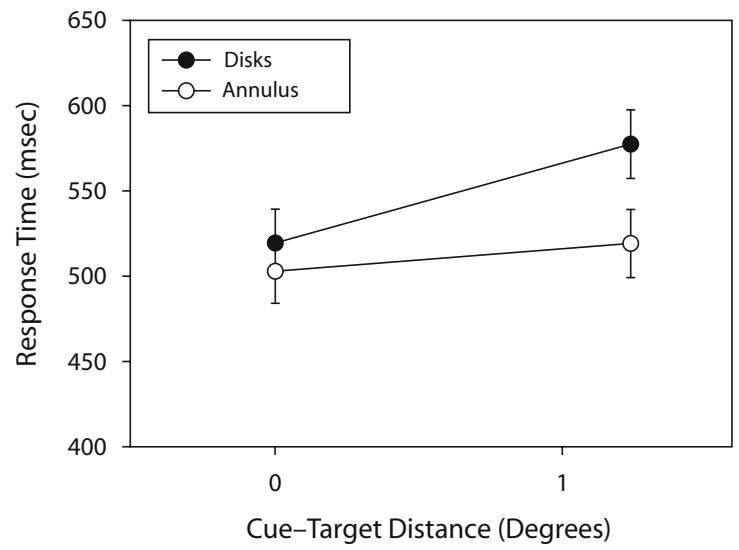

Figure 6. Mean response times in Experiment 3 shown as a function of cue-to-target distance. Error bars indicate the standard errors of the means. 
dition ( 0 cue-target distance) did not differ across the array and annulus conditions $[t(19)=1.59$, n.s.]. Thus, the difference in the size of the two effects seems to derive from a greater slowing in the one-away condition, relative to the valid condition, for the disks displays, as compared with the annulus displays, implying that the disks allowed the observers to focus their attention more precisely than they could without the supporting disks.

The results of this experiment reduce the concern that the disks were irrelevant to the cuing effects that were observed in Experiment 2. In the final experiment, we explored the comparability of the cuing task with the walk task further by asking whether an endogenous cue could similarly direct attention within an array of disks that is too dense to support a reliable attentional walk.

\section{EXPERIMENT 4}

Experiment 2 demonstrated that attention can be allocated on the basis of an exogenous cue within arrays that are sufficiently dense as to yield chance performance in the attentional walk task, and Experiment 3 confirmed that this was not because the disks were irrelevant for the cuing task. This final experiment tested whether attention can be allocated within dense arrays on the basis of an endogenous cue. The reason for testing this possibility is that all the shifts of attention in the attentional walk task other than the first shift to the start disk are cued endogenously, usually through auditory tones indicating the shift of attention to an adjacent disk. Therefore, it is possible that the attentional walk task does reflect limitations of attentional resolution but that it is the resolution of endogenously controlled attention that is critical, and all that we have probed so far with the cuing measure has been exogenously controlled attention. It is possible, even likely, that the resolution of exogenously and endogenously controlled attention differs.

Displays consisted of coarse and dense arrays of disks like those used in Experiment 2. A single item within the array was cued endogenously using the following method. An arrow was presented at fixation that pointed to one of four locations within the array $\left(45^{\circ}, 135^{\circ}, 225^{\circ}\right.$, or $315^{\circ}$ ). The observers were asked to attend to the disk that was opposite that location, reflected across the horizontal meridian (see Figure 7). This somewhat elaborate cuing technique was used in order to be able to specify a single disk within a dense array without placing an excessive load on working memory (e.g., assigning each location a letter or color) and yet avoiding exogenous contributions that can occur when an arrow points directly to the cued location, such as through colinearity and/or apparent motion.

\section{Method}

Except where noted here, the method was the same as that in Experiment 2

Observers. Twenty individuals from the same pool as that in Experiment 2 were tested. None had participated in Experiment 2, and all reported normal or corrected-to-normal visual acuity.

Stimuli. The arrays were identical to those in Experiment 2, except that the cue was a central arrow that was $0.54^{\circ}$ from tip to tail.
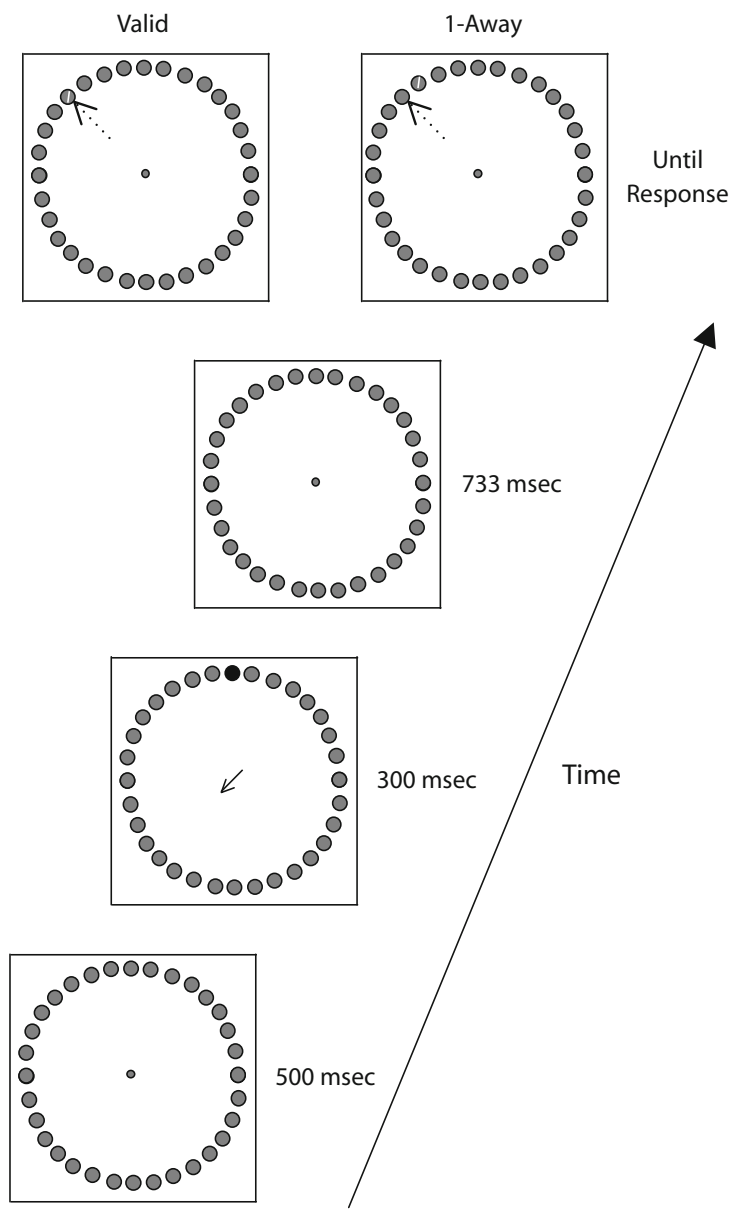

Figure 7. Experiment 4 trial events. A central arrow indicated the target location indirectly. The cue pointed to a location, indicating that the target had an $80 \%$ chance of appearing at the location that was in the position opposite to that location, reflected across the horizontal meridian. The top row illustrates alternative response displays for the two validity conditions. The arrows in these displays were not present during the experiment. They are shown here to indicate the cued location.

The line segments forming the arrow head extended $0.22^{\circ}$ from the tip at a $15^{\circ}$ angle relative to the shaft. The arrow was centered on the screen and pointed at one of the following four locations: $45^{\circ}, 135^{\circ}$, $225^{\circ}$, or $315^{\circ}$.

Task. The task was the same as that in Experiment 2: Report the orientation of the line segment that appears at the end of each trial as quickly as possible while maintaining at least $95 \%$ accuracy.

Design. A 2 (density: coarse or dense) $\times 2$ (validity: valid or one-away) within-subjects design was used. Unlike in Experiment 2, there were nonequal probabilities associated with each validity condition. Specifically, the target occurred in the valid location $80 \%$ of the time and in each of the two one-away positions (to the left or right of the cued disk) $10 \%$ of the time. This was done to encourage the use of the cue. This validity manipulation also accounts for the reduced design (i.e., no two-away conditions). In order to obtain a sufficient number of observations in the lower probability conditions, the number of conditions was reduced.

Procedure. Each session began with a set of written instructions presented on the computer monitor. These were reinforced with a 
figure on a piece of paper that helped describe the task. The observers were shown examples of each of the four possible cuing conditions, using dense-array displays as examples. They were oriented to the horizontal meridian and were shown what it meant for it to be indicating the position opposite the location toward which the arrow pointed, reflected across the horizontal meridian. The observers then participated in a full block of 40 trials of practice and were given the opportunity to ask questions regarding the task. The written instructions emphasized that the target would appear in the cued location more often than not and that they should use the cue to help them find and report the orientation of the target line more quickly than they might otherwise.

Trial events were identical to those in Experiment 2, except that the arrow cue occurred instead of the flash cue, the duration of the arrow was $300 \mathrm{msec}$, and the time between the offset of the arrow and the onset of the target was $733 \mathrm{msec}$.

\section{Results and Discussion}

Mean RTs are shown in Figure 8 as a function of the spatial separation between the cue and the target. ERs are given in Table 1. The RT data were submitted to a 2 (density) $\times 2$ (validity) ANOVA. There was a reliable main effect of validity $[F(1,19)=25.85, p<.001]$ but no reliable effect of density $[F(1,19)=2.93$, n.s.]. Planned comparisons confirmed that there was a reliable cuing effect for both display conditions [dense, $32 \mathrm{msec}, t(19)=$ $2.6, p<.05$; coarse, $85 \mathrm{msec}, t(19)=5.7, p<.001]$ A significant interaction between density and validity $[F(1,19)=10.76, p<.01]$ confirmed that the validity effect was larger in the coarse condition than in the dense condition. All of the same analyses were conducted on the ERs. No effects that were in a direction different from those in the RT data were found.

The results show that attention can be allocated to an individual item within dense arrays that fail to support reliable attentional walks even when attention is cued endogenously. As in Experiment 2, this indicates that failures in the attentional walk task cannot be attributed solely to limitations in the spatial extent of attentional selection (attentional resolution). Rather, it suggests that failures in the attentional walk task also reflect the preci-

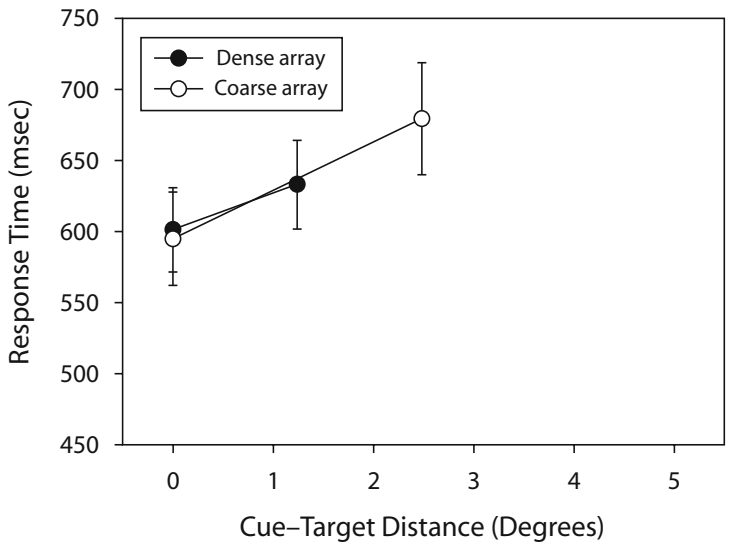

Figure 8. Mean response times in Experiment 4 shown as a function of cue-to-target distance. Error bars indicate the standard errors of the means. sion with which attention can be localized within these arrays.

\section{GENERAL DISCUSSION}

The experiments reported here emphasize that there are multiple limitations of visual attention that need to be considered when characterizing the spatial extent of attention. In particular, they suggest that the attentional walk task, which was developed as a method for measuring attentional resolution, also reflects limitations of the precision with which attention can be localized and that this limitation may even dominate performance in that particular task. To be clear, the claim here is that the smallest densities for which observers can reliably execute an attentional walk do not necessarily reflect the smallest "window" of attention that can be achieved under those conditions. If it did, there should have been no cuing advantage for valid targets over one-away targets in dense displays in Experiments 2-4 of the present study. Instead, we suggest that it reflects some combination of a minimal "window size" and the precision with which the "window" can be focused on a given targeted location/item.

An important difference between the attentional walk task and the cuing task is that the attentional walk task requires reliable navigation from an endogenously maintained starting point (i.e., the current focus of attention) to an endogenously maintained target point (i.e., the next disk in the direction that is indicated by the current command). If either one of those representations is disrupted, the walk can fail. The fact that all of the disks are identical in the standard attentional walk task may contribute to the difficulty in maintaining those landmarks. In particular, it forces observers to rely on spatial location more than they might if the items differed in other attributes as well (e.g., color). Consistent with this possibility, we have found that attentional walks can be executed more reliably in dense arrays when the disks are heterogeneously colored than when they are identical (Moore, Lanagan-Leitzel, Chen, Halterman, \& Fine, 2007).

Another difference between cuing tasks and the attentional walk is that the attentional walk task requires multiple shifts of attention, whereas the cuing tasks used here required only one shift to the cued item. In an experiment not reported here, we found a one-away cuing effect in dense arrays following multiple exogenous cues timed to match the steps in an attentional walk task (Lanagan $\&$ Moore, 2003). Although this reduces the differences across tasks, it is difficult to compare this with multiple shifts in the attentional walk task, because with multiple exogenous cues, the final cue may be the only one that matters. This follows because it is not necessary for observers to successfully maintain the location of the previous focus of attention to make the next shift if it is explicitly cued. Ongoing work is being done to investigate the contribution of individual steps within the attentional walk task to overall performance.

Yet another difference between cuing tasks and the attentional walk task is that more complete selection may be required to succeed in the attentional walk task than 
is needed for producing a cuing effect. Although the oneaway cuing effect does indicate that the cued item was processed selectively, relative to one item away, it does not necessarily indicate that the cued item was selected to the exclusion of other items. It is possible that the walk task requires exclusive selection to succeed, whereas the cuing task requires only partial selection. Assuming that is true, however, it begs the question as to why there is this difference. One reason could be that controlling a shift of attention from one endogenously maintained item/location to another endogenously maintained item/location requires exclusive selection because any "leakage" from another object/location would disrupt the representation of one or the other of the endpoints of the shift. Thus, the difficulty of the walk task would reflect the precision with which attention can be controlled.

All of these differences between the cuing task and the attentional walk task highlight the difficulties of drawing conclusions based on comparisons of performance across different tasks. In a recent study, we have begun to explore the distinction between limitations in performance due to the pooling of information across a minimal selection area (i.e., attentional resolution) and limitations in performance due to missampling of information caused by imprecise targeting (i.e., the precision with which attention can be localized), using a single task and more formal modeling methods (Palmer \& Moore, in press). In particular, we adapted psychophysical theories of contrast sensitivity to a spatial cuing situation in which nontarget stimuli compete with targets for controlling behavior. Doing so demonstrated that the two mechanisms - pooling versus imprecise targeting - yield distinct predictions. In experiments in which attention was focused narrowly, the results were consistent with an imprecise-targeting model and were inconsistent with a pooling model (for discussions of similar mechanisms, see Bahcall \& Kowler, 1999; Strasburger, 2005). In contrast, when attention was focused more broadly, the results were consistent with a pooling model (Yigit, Palmer, \& Moore, 2008). These methods have not yet been extended to situations in which there are many nearby identical stimuli, such as in this study and with the attentional walk task more generally. It is possible that under those conditions, imprecise targeting is the main limit on performance even for partially valid cuing situations. This is a direction for further inquiry.

An implication of the conclusions drawn from the present study is that they suggest a qualification of some of the conclusions drawn by Intriligator and Cavanagh (2001). In particular, a focus of their discussion was the extreme dissociation between two-point acuity and attentional resolution as estimated using the attentional walk task. In particular, it was emphasized that "the very smallest region that can be selected is much larger than the smallest detail that can be seen" (Intriligator \& Cavanagh, 2001, p. 203). The present results suggest that it is not necessarily a dissociation between the smallest region that can be selected and the smallest visible detail that is revealed through the walk task; a selective region can be set smaller than what would be suggested by the attentional walk task. Instead, the dissociation may be between the finest level of preci- sion with which a shift of attention from an endogenously maintained starting point to an endogenously maintained target can be made and the smallest visible detail. These are not constructs that are as intuitively comparable, but they nonetheless seem to be the ones that contrast with each other.

In summary, the present results demonstrate that individual items can be processed selectively, relative to immediately adjacent items, even in arrays in which items are separated by as little as $1.2^{\circ}$. These densities do not support reliable attentional walks. Therefore, we suggest that attentional resolution and the precision with which attention can be localized are separate limitations and that the attentional walk task is better suited for revealing the limitations of attentional localization than those of attentional resolution.

\section{AUTHOR NOTE}

This work was supported by NIH Grant MH067793 to C.M.M. The authors are grateful to Elisabeth Hein, J. Toby Mordkoff, and John Palmer for helpful discussions concerning this and related work. Correspondence concerning this article should be addressed to C. M. Moore, Department of Psychology, E11 Seashore Hall, University of Iowa, Iowa City, IA 52242 (e-mail: cathleen-moore@uiowa.edu).

\section{REFERENCES}

BAHCALl, D. O., \& Kowler, E. (1999). Attentional interference at small spatial separations. Vision Research, 39, 71-86.

Cutzu, F., \& Tsotsos, J. K. (2003). The selective tuning model of attention: Psychophysical evidence for a suppressive annulus around an attended item. Vision Research, 43, 205-219.

Downing, C. J., \& Pinker, S. (1985). The spatial structure of visual attention. In M. I. Posner \& O. S. M. Marin (Eds.), Attention and performance XI (pp. 171-187). Hillsdale, NJ: Erlbaum.

ERIKSEN, C. W., \& Hoffman, J. E. (1972). Temporal and spatial characteristics of selective encoding from visual displays. Perception \& Psychophysics, 12, 201-204.

ERIKSEN, C. W., \& ST. James, J. D. (1986). Visual attention within and around the field of focal attention: A zoom lens model. Perception \& Psychophysics, 40, 225-240.

ERIKSEN, C. W., \& YEH, Y. Y. (1985). Allocation of attention in the visual field. Journal of Experimental Psychology: Human Perception \& Performance, 11, 583-597.

Fernandez-Duque, D., \& Johnson, M. L. (1999). Attention metaphors: How metaphors guide the cognitive psychology of attention. Cognitive Science, 23, 83-116.

He, S., Cavanagh, P., \& Intriligator, J. (1996). Attentional resolution and the locus of visual awareness. Nature, 383, 334-337.

He, S., Cavanagh, P., \& Intriligator, J. (1997). Attentional resolution. Trends in Cognitive Sciences, 1, 115-121.

Henderson, J. M., \& Macquistan, A. D. (1993). The spatial distribution of attention following an exogenous cue. Perception \& Psychophysics, 53, 221-230.

Intriligator, J., \& CaVAnagh, P. (2001). The spatial resolution of attention. Cognitive Psychology, 43, 171-216.

Juola, J. F., Bouwhuis, D. G., Cooper, E. E., \& Warner, C. B. (1991). Control of attention around the fovea. Journal of Experimental Psychology: Human Perception \& Performance, 17, 125-141.

LABERGE, D. (1983). Spatial extent of attention to letters and words. Journal of Experimental Psychology: Human Perception \& Performance, 9, 371-379.

LABERge, D., \& Brown, V. (1986). Variations in size of the visual field in which targets are presented: An attentional range effect. Perception \& Psychophysics, 40, 188-200.

LaBerge, D., \& Brown, V. (1989). Theory of attentional operations in shape identification. Psychological Review, 96, 101-124.

LanaGan, L. K., \& Moore, C. M. (2003). Contrasting the resolution of exogenously and endogenously controlled attention [Abstract]. 
Journal of Vision, 3, 328a. Available at journalofvision.org/3/9/328/, doi: $10.1167 / 3.9 .328$

Moore, C. M., Lanagan-Leitzel, L. K., Chen, P., Halterman, R., $\&$ Fine, E. M. (2007). Nonspatial attributes of stimuli can influence spatial limitations of attentional control. Perception \& Psychophysics, 69, 363-371.

MounTs, J. R. W. (2000a). Attentional capture by abrupt onsets and feature singletons produces inhibitory surrounds. Perception \& Psychophysics, 62, 1485-1493.

MounTs, J. R. W. (2000b). Evidence for suppressive mechanisms in attentional selection: Feature singletons produce inhibitory surrounds. Perception \& Psychophysics, 62, 969-983.

Palmer, J., \& Moore, C. M. (in press). Using a filtering task to measure the spatial extent of selective attention. Vision Research.

Posner, M. I. (1980). Orienting of attention. Quarterly Journal of Experimental Psychology, 32, 3-25.

Posner, M. I., Snyder, C. R. R., \& Davidson, B. J. (1980). Attention and the detection of signals. Journal of Experimental Psychology: General, 109, 160-174.

SAGI, D., \& Julesz, B. (1986). Enhanced detection in the aperture of focal attention during simple discrimination tasks. Nature, 321, 693-695.

Steinman, B. A., Steinman, S. B., \& Lehmkuhle, S. (1995). Visual attention mechanisms show a center-surround organization. Vision Research, 35, 1859-1869.

Strasburger, H. (2005). Unfocussed spatial attention underlies the crowding effect in indirect form vision. Journal of Vision, 5, 1024-1037. Available at journalofvision.org/5/11/8/, doi:10.1167/5.11.8.

Tsotsos, J. K., Culhane, S. M., WaI, W. Y. K., LaI, Y. H., Davis, N., \& Nuflo, F. (1995). Modeling visual attention via selective tuning. Artificial Intelligence, 78, 507-545.

Yigit, S., Palmer, J., \& Moore, C. M. (2008, May). Partially valid cueing and spatial filtering reveal different kinds of selection. Poster presented at the 8th Annual Meeting of the Vision Sciences Society, Naples, FL.

\section{NOTES}

1. Arcs of $140^{\circ}$ were used in the first experiment because we were replicating the design of Intriligator and Cavanagh (2001). More recently, we have been using full arrays to reduce grouping cues.

2. Because RT is the main dependent measure and the observers were instructed to make their responses as quickly as possible while maintaining a high level of accuracy, the purpose of the ER analyses was to provide assurance against drawing conclusions from patterns in the RT data that could have been due to a speed-accuracy trade-off. We therefore present $F$ and $t$ values for the ER analyses only when there was a significant effect that was not there in the RTs or that was in the direction opposite to that in the RTs.

(Manuscript received February 22, 2005; revision accepted for publication November 12, 2007.) 